\title{
Relating the Progeny Production Curve to the Speed of an Epidemic
}

\author{
Francis J. Ferrandino
}

Associate Scientist, Department of Plant Pathology and Ecology, The Connecticut Agricultural Experiment Station, P.O. Box 1106, New Haven 06504.

Accepted for publication 13 October 2012.

\section{ABSTRACT}

Ferrandino, F. J. 2013. Relating the progeny production curve to the speed of an epidemic. Phytopathology 103:204-215.

The dependence of the initial infection rate, $r$, on the basic reproductive number, $R_{0}$, and the temporal moments of the progeny production curve are examined. A solution to the linearized Kermack-McKendrick equation is presented and used to analyze a variety of theoretical models of pathogen reproduction. The solution yields a relation between $r$ and the basic reproductive number, $R_{0}$; the mean time between pathogen generations, $\mu$; and the standard deviation about this mean, $\sigma$. A transformation using the dimensionless variables $r \mu$ and $r \sigma$ is introduced, which maps the solution onto a one-dimensional curve. An approximation for the value of $r$ in terms of $R_{0}$ and the first four temporal moments of the reproductive curve is derived. This allows direct comparison of epidemics resulting from theoretical models with those generated using experimentally obtained reproduction curves. For epidemics characterized by a value of $r \mu<5$, the value of $r$ is well determined $(<2 \%)$ by this fourth-order expansion regardless of the functional form of the reproduction curve.

Additional keywords: Laplace Transform, sporulation curve.
A large range of mathematical models of plant disease epidemics have been shown $(34,52)$ to be special cases of the more general Kermack-McKendrick epidemic model (KM model) (31). This model is based on the assumption that the reproductive behavior of plant disease lesions is simply a function of lesion age, $\tau$. The KM model, in turn, is based on the mathematical model of population growth presented by Lotka (14,36-41).

The basic assumption of the KM model is that the reproductive capacity of an infected population at time $t$ due to individuals infected at time $t-\tau$ is expressible as the product of the number of individuals entering the population at time $t-\tau$, the per capita fecundity at age $\tau,[m(\tau)$, the number of daughter lesions per mother lesion per unit time in a totally susceptible population], and the probability of survival to age $\tau, l(\tau)$. The product of the last two terms is represented by the reproduction curve, $\Re(\tau)$ (where $\tau$ is the age of the mother lesion), defined as the number of daughter lesions produced per mother lesion per unit time, and includes the effects of latency, the rate of daughter lesion production over the infectious period, and the removal of infectious tissue from the system by senescence or defoliation of the host tissue. The effect of age of the host tissue on infectivity is ignored (20). The KM model can be expressed as

$$
\begin{aligned}
& \frac{d I(t)}{d t}=\frac{H(t)}{H_{\max }} \cdot \int_{0}^{t} \frac{d I(t-\tau)}{d t} \cdot \Re(\tau) d \tau+g(t) \\
& H_{\max }=H(t)+I(t) \\
& I(t \leq 0)=g(t<0)=0
\end{aligned}
$$

where $H(t)$ is the amount of healthy susceptible tissue at time $t$, $H_{\max }$ is the total amount of susceptible plant tissue, $I(t)$ is the

Corresponding author: F. J. Ferrandino;

E-mail address: francis.ferrandino@po.state.ct.us

http://dx.doi.org/10.1094/PHYTO-04-12-0093-R

(C) 2013 The American Phytopathological Society amount of infected tissue (including latent, $L$; sporulating, $S$; and removed, $R$ ) at time $t$, and $g(t)$ is the rate of new lesion creation at time $t$ due to sources outside our system. The function $g(t)$ is necessary to start the epidemic and represents the effects of local initial inoculum that usually overwinters as well as inoculum blown in from a distance. For simplicity, in what follows, I will ignore the effect of crop growth $(4,16,17,19,22,23,32,59)$. The units of $H(t)$, $H_{\max }$, and $I(t)$ and the product $\mathrm{g}(t) d t$ can be lesions, area, or density (area per ground area), as long as they are consistent (43). The initial quotient on the right side of equation 1 is the so-called "correction factor" (57), which accounts for the effect of multiple infection. In the early stages of the epidemic, $I<<H_{\max }$ and $H(t) \cong$ $H_{\max }$, so that the correction factor is approximately equal to one. In this regime, equation 1 is linearized. In addition, assuming that the function $g(t)$ is a spike function at $t=0$ with area $I_{0}[g(t)=$ $I_{0} \delta(t) ; \delta(t)$ is the Dirac $\delta$ function] (13), then the asymptotic solution of equation 1 is an increasing exponential in time such that (see Appendix in 14 written by A. J. Lotka):

$$
I(t)=I_{0} \cdot \exp (r t)
$$

where $r$, what I will call the initial infection rate (Vanderplank's $r_{l}$, the logarithmic rate of disease increase [58]; Metz and Deikman call $r$ "the intrinsic rate of natural increase" (47, page 145) is the real-valued root of the following equation

$$
1=\int_{0}^{\infty} \exp \left(-r t^{\prime}\right) \cdot \Re\left(t^{\prime}\right) d t^{\prime}
$$

Equation $2 \mathrm{~b}$ is known as the characteristic equation of equation 1 (47, page 145). The integral in equation $2 b$ is, by definition, the Laplace Transform of $\Re(\tau)$ (Appendix) and, as Metz and Deikman noted, the Laplace Transform of the reproduction function, evaluated at $r$, has a value of unity (47, page145):

$$
1=\int_{0}^{\infty} \exp \left(-r t^{\prime}\right) \cdot \mathfrak{R}\left(t^{\prime}\right) d t^{\prime}=\widetilde{\mathfrak{R}}(r)
$$

where the tilde represents the Laplace Transform (see Appendix). 
Equations $2 \mathrm{a}$ and $2 \mathrm{~b}$ or, equivalently, $2 \mathrm{~b}^{\prime}$ specify a solution to equation 1 during the early stages of an epidemic, when $H(t) \cong$ $H_{\max }$. This solution (30) is characterized by a stable lesion age probability distribution, $c(\tau)$, such that the product $c(\tau) \cdot I(t) \mathrm{d} \tau$ is the number of lesions at time, $t$, between the ages of $\tau$ and $\tau+\mathrm{d} \tau$, and is given by

$$
c(\tau)=r \cdot \exp (-r \tau)
$$

We seek the relation between the shape of the reproduction curve, $\Re(\tau)$, the value of $r$, and the resultant disease progress curve, $I(t)$. The natural time scales for the reproduction curve, $\Re(\tau)$, can be obtained from its temporal moments. Following the notation of Dublin and Lotka (14), I define $R_{\mathrm{n}}$ to be the $n$th order temporal moment of $\Re(\tau)$ about $\tau=0$, such that

$$
R_{n}=\int_{0}^{\infty} \tau^{n} \cdot \Re(\tau) d \tau=\left.(-1)^{n} \frac{\partial^{n} \widetilde{\Re}(\xi)}{\partial \xi^{n}}\right|_{\xi=0}
$$

where $n$ takes on integral values $(0,1,2,3, \ldots)$. The expression on the far right side of equation 4 is a very useful property of the Laplace Transform and is used throughout this article to evaluate the temporal moments of reproduction curves (33). Of course, for $n=0$, the integral in equation $4, R_{0}$ (12) the basic reproductive ratio), is the average total number of daughter lesions per mother lesion throughout the infectious stage (25). For $n=1$, equation 4 yields the mean time between generations of the pathogen, $\mu=$ $R_{1} / R_{0}$. In general, the $n$ th-order temporal moment of $\mathfrak{R}(\tau)$ about $\tau=0, m_{\mathrm{n}}{ }^{\prime}$, can be used to generate the centered moments (about $\tau=$ $\mu), m_{\mathrm{n}}$; which, in turn, can be nondimensionalized (15) (Table 1).

Once the reproduction curve, $\Re(\tau)$, is known (assumed or measured), epidemics following the above equations can be easily calculated. Segarra et al. (52) have shown that the Vanderplank (58) and compartmental models (1-3,10,21,26-28,42,49,50, $59,60)$ fit into the above scheme. Leonard and Mundt (34) solved equation $2 \mathrm{~b}$ using a triangular form $(35,45)$ for the reproduction curve to describe a number of plant-pathogen systems for which sporulation curves had been measured $(5-8,11,29,46,48,51,53)$. Van den Bosch et al. (54-56) suggested using a delayed $\gamma$ distribution function for the reproduction curve. Segarra et al. (52) (Fig. 1, caption) declared that the proper way to compare various models was to assure that "The average duration of the infection cycle is $p+i \ldots$ " for all distributions under consideration. In the above statement, $p$ is the latent period and $i$ is the infectious period. Cunniffe et al. (9) used this prescription to compare a number of different models. As I will demonstrate, due to differences in the way the above models are constructed, this condition results in model comparisons, which have differing values for $\mu$ and $\sigma$, the first two moments of the progeny production curve and, thus, are not directly comparable (18).

My goal in this article is to compare and contrast the resulting disease progress curves for all these epidemic models under the constraint that the basic reproductive ratio $\left(R_{0}\right)$, the mean time between successive generations $\left(\mu=R_{1} / R_{0}\right)$, and the standard deviation $\left\{\sigma=\left[R_{2} / R_{0}-\left(R_{1} / R_{0}\right)^{2}\right]\right\}$ about this mean are identical. The first two temporal moments of the reproduction curve are directly obtainable from the reproduction curve or its Laplace Transform either by direct integration or via the evaluation of the derivatives of the Laplace Transform (equation 4). This allows the direct comparison of many different progeny production models with epidemics generated using experimentally determined reproduction curves. Nondimensionalized equations using these two time scales result in a simpler exposition of the parameters governing the resulting epidemic. A comparison of a number of the above forms for $\Re(\tau)$ are illustrated in Figure 1 and discussed in detail in the next section.

\section{THEORY AND APPROACHES}

Theoretical models of progeny production start with a proposed functional form of the time rate of "daughter lesion" production as a function of "mother lesion" age, These curves are characterized by specific model-dependent parameters and the integrated area under these curves is by definition $R_{0}(12)$, the basic reproductive ratio. In what follows, I successively discuss each model of progeny production in terms of its natural parameters. Then, the Laplace Transform of the specific reproduction curve is presented and expressed in terms of its first two temporal moments ( $\mu$ and $\sigma$ ). In this form, the models can be directly compared.

The comparison of differently shaped reproduction curves. Vanderplank's square pulse of inoculum. Vanderplank (58) put forward a relatively simple epidemic model based on a square pulse (SP) of inoculum (Fig. 1), such that

$$
\Re_{S P}(\tau, p, i)=\left\{\begin{array}{cc}
0 & \tau<p \\
\frac{R_{0}}{i} & p \leq \tau \leq p+i \\
0 & p+i<\tau
\end{array}\right.
$$

TABLE 1 . Temporal moments of the reproduction curve $\Re(\tau)$ in terms of $R_{\mathrm{n}}{ }^{a}$

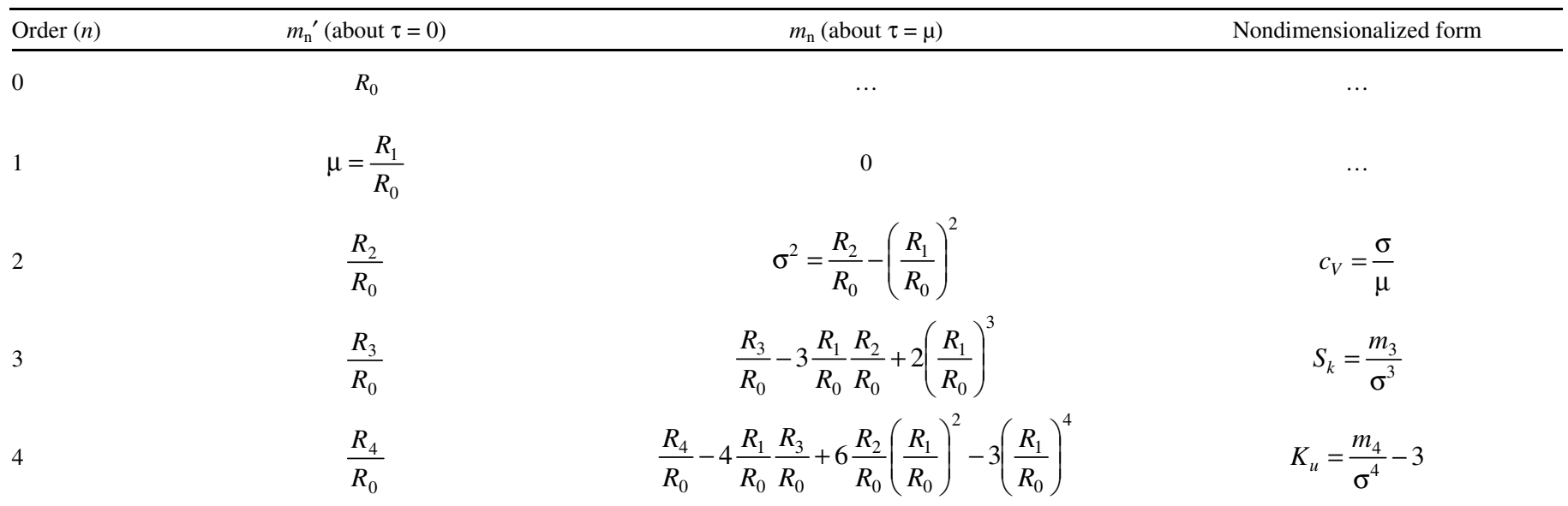

a Defined by equation 4, repeated here for clarity: $R_{n}=\int_{0}^{\infty} \tau^{n} \cdot \Re(\tau) d \tau=\left.(-1)^{n} \frac{\partial^{n} \mathfrak{\Re}(\xi)}{\partial \xi^{n}}\right|_{\xi=0}$. The nondimensional forms of the second-, third-, and fourthorder centered moments are the coefficient of variation $\left(c_{\mathrm{V}}\right)$, the skewness $\left(S_{\mathrm{k}}\right)$, and the excess kurtosis $\left(K_{\mathrm{u}}\right)$, defined here. 
where $p$ is the latent period (the time delay before infected tissue becomes infectious) and $i$ is the infectious period (the time span during which a mother lesion produces $R_{0}$ daughter lesions). Thus, the progeny production rate per unit time is assumed to be a constant given by the value of the quotient $R_{0} / i$. By definition (Table 1), the mean time between infection and the production of secondary infections for $\Re_{S P}(\tau)$ is given by $\mu=p+i / 2$ and the standard deviation about this mean, $\sigma=i /(12)^{1 / 2}$ (Table 2$)$. The coefficient of variation $\left(c_{\mathrm{V}}=\sigma / \mu\right)$ (Table 1$)$ of equation 8 can vary from $0(i<<p)$ to a maximum value of $3^{-1 / 2} \approx 0.577$.

If equation 5 is inserted into equation $2 b^{\prime}$, the result is

$$
1=\left.\widetilde{\mathfrak{R}}_{S P}(\xi)\right|_{\xi=r}=R_{0} \frac{e^{-r p}-e^{-r(p+i)}}{r i}=R_{0} e^{-r \mu} \frac{\sinh (\sqrt{3} r \sigma)}{\sqrt{3} r \sigma}
$$

Note that the term on the far right side of equation 6 is expressible as a function of $r \mu$ times a function of $r \sigma$. In fact, the function of $r \mu$ (i.e., $R_{0} e^{-r \mu}$ ) has a very special meaning, as discussed below.

Consider the limiting case of equations 5 and 6 as the infectious period, $i$, becomes very short. The reproduction curve becomes a Dirac $\delta$ function $\delta\left(\mu_{\delta}\right)(13)$, such that all $R_{0}$ of the daughter lesions are produced in perfect synchrony exactly $\mu_{\delta}$ days after the mother lesion was infected. For this special case, equation 6 yields

$$
\mathfrak{R}_{\delta}(\tau)=R_{0} \delta\left(\mu_{\delta}\right) \Rightarrow 1=\left.\widetilde{\mathfrak{R}}_{\delta}(\xi)\right|_{\xi=r_{\delta}}=R_{0} e^{-r_{\delta} \mu_{\delta}} \Rightarrow r_{\delta}=\frac{\ln \left(R_{0}\right)}{\mu_{\delta}}
$$

where the subscripted $\delta$ signifies that the reproduction curve is given by the Dirac $\delta$ function (13).

Delayed $\gamma$ distribution. In an attempt to have a more realistic, smoothly varying reproduction curve, van den Bosch et al. (5456) suggested using a time-delayed $\gamma$ distribution to describe the reproduction curve (Fig. 1):

$$
\Re_{\Gamma}\left(\tau, n, \tau_{D}, \lambda\right)=\left\{\begin{array}{cc}
0 & \tau<\tau_{D} \\
\frac{R_{0} \lambda^{n}\left(\tau-\tau_{D}\right)^{n-1} e^{-\lambda \tau}}{\Gamma(n)} & \tau \geq \tau_{D}
\end{array}\right.
$$

where $\Gamma(n)$ is the $\gamma$ function of order $n$ (for integral $n, \Gamma(n)=(n-$ $1)$ !) and $\lambda$ is a rate parameter. When equation 8 is inserted into equation $2 b$, the result is

$$
1=\left.\widetilde{\mathfrak{R}}_{\Gamma}(\xi)\right|_{\xi=r}=R_{0} e^{-r \tau} D \lambda^{n}(r+\lambda)^{-n}=R_{0} e^{-r \mu} e^{r \sigma \sqrt{n}}\left(1+\frac{r \sigma}{\sqrt{n}}\right)^{-n}
$$

where $\mu=\tau_{D}+n / \lambda$ and $\sigma^{2}=n / \lambda^{2}$ (Table 2). The $c_{\mathrm{V}}$ (Table 1) of equation 9 can vary from $0(\sigma<<\mu)$ to a maximum value of $n^{-1 / 2}$ for $\tau_{D}=0$. Once again, the right side of equation 9 is expressible as a function of $r \mu$ times a function of $r \sigma$. In the limit as $n$ and $\lambda$ approach infinity, holding the ratio $n / \lambda$ constant, the right side of equation 9 approaches the Dirac $\delta$ value (i.e., $R_{0} e^{-r \mu}$; equation 7 ) as $\sigma$ goes to zero.

Lewontin's triangular pulse. Lewontin (35) suggested a triangular form for the reproduction curve:

$\Re_{L}\left(\tau, \tau_{D}, \tau_{i}, f\right)=\left\{\begin{array}{cc}0 & \tau<\tau_{D} \\ \frac{2 R_{0}}{f \tau_{i}^{2}}\left(\tau-\tau_{D}\right) & \tau_{D} \leq \tau \leq \tau_{D}+f \cdot \tau_{i} \\ \frac{2 R_{0}}{(1-f) \tau_{i}^{2}}\left(\tau_{D}+\tau_{i}-\tau\right) & \tau_{D}+f \cdot \tau_{i} \leq \tau \leq \tau_{D}+\tau_{i} \\ 0 & \tau>\tau_{D}+\tau_{i}\end{array}\right.$

where $\Re_{L}(\tau)$ takes the form of a triangle starting at $\tau_{D}$ (delay time) with a base of width $\tau_{i}$ (infectious period) and its apex located at $\tau=\tau_{D}+f \tau_{i}$. The parameter $f$ can vary from 0 to 1 . A value of $f=0$ corresponds to a right triangle skewed toward the right, $f=0.5$

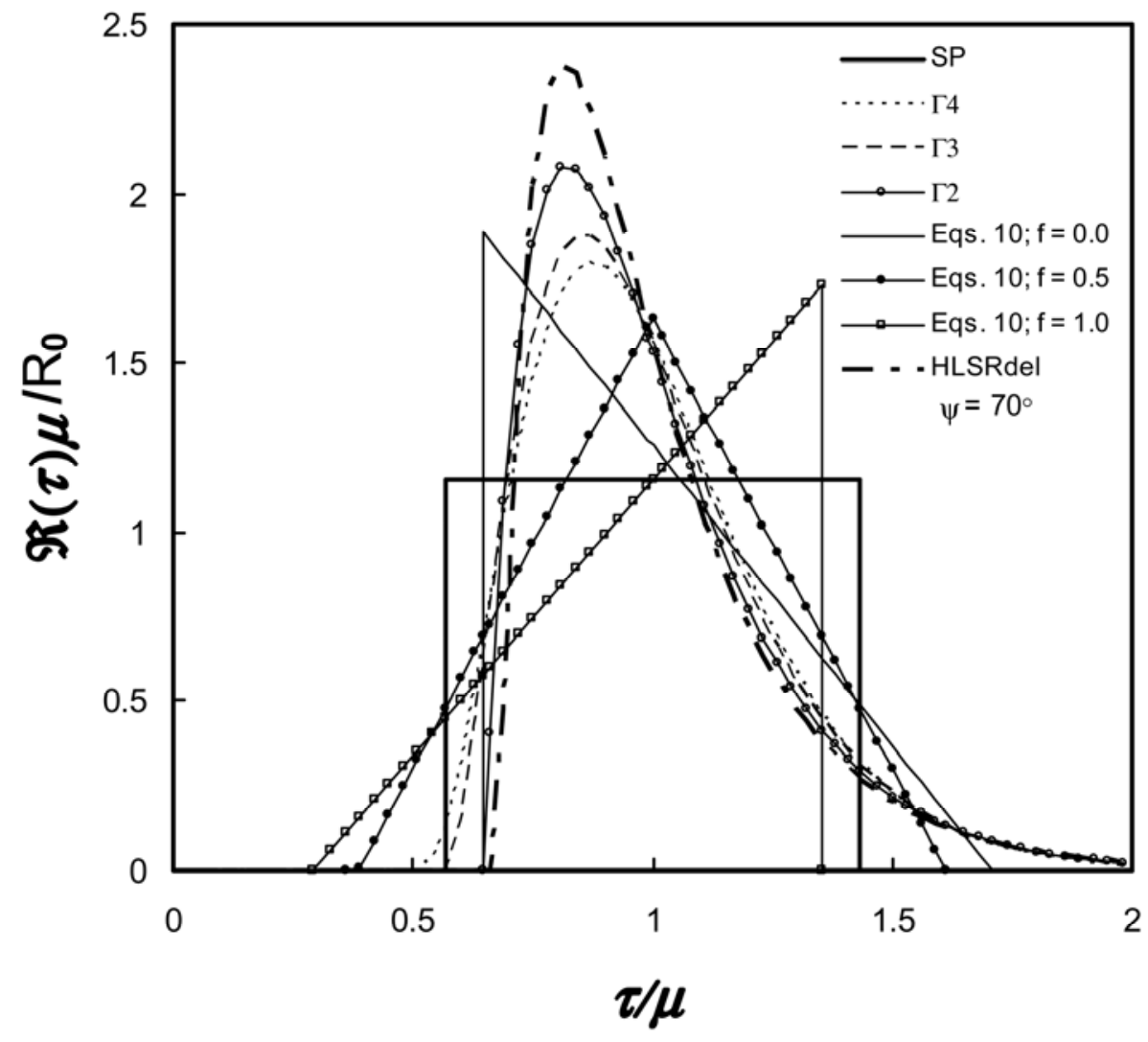

Fig. 1. Comparison of the various reproductive curves discussed in the text. All curves have unit area, the same mean, $\mu$, and the same coefficient of variation $\left(c_{\mathrm{V}}=0.25\right)$ 
yields an isosceles triangle, and $f=1.0$ corresponds to a right triangle skewed toward the left (Fig. 1). When equation 10 is inserted into equation $2 \mathrm{~b}$, the result is

$$
1=\left.\widetilde{\mathfrak{R}}_{L}(\xi)\right|_{\xi=r}=\frac{2 R_{0}}{r^{2} \tau_{i}^{2} f(1-f)}\left[\begin{array}{c}
(1-f)\left(e^{-r \tau_{D}}-e^{-r\left(\tau_{D}+f \tau_{i}\right)}\right)- \\
f\left(e^{-r\left(\tau_{D}+f \tau_{i}\right)}-e^{-r\left(\tau_{D}+\tau_{i}\right)}\right)
\end{array}\right]
$$

The moments of $\Re_{L}(\tau)$ are given in Table 2. The $c_{\mathrm{V}}$ (Table 1 ) of equation 9 can vary from $0(\sigma<<\mu)$ to a maximum value of $2^{-1 / 2} \approx$ 0.707 for $\tau_{D}=0$ and $f=0$. Although a bit more algebraically challenging, once again, the right side of equation 11 approaches the Dirac $\delta$ value (i.e., $R_{0} e^{-\mathrm{r \mu}}$; equation 10 ) as $\sigma^{2}$ goes to zero (Appendix) and is expressible as a function of $r \mu$ times a function of $r \sigma$ (equation A7).

Compartmental models. HLSR model. Compartmental models are often used in theoretical epidemiology. Infection sites are classified as being either healthy $(H)$, latent $(L)$, infectious $(S)$, or postinfectious $(R)$ (52). The total amount of infected tissue $(I)$ includes latent $(L)$, sporulating $(S)$, and postinfectious or removed $(R)$. This corresponds to the more usual terminology of susceptible $(S)$, exposed $(E)$, infectious $(I)$, and removed $(R)$ used in human and animal epidemiology. These models are formulated as systems of linked differential equations $(10,26,49)$ :

$$
\begin{aligned}
& -\frac{d H}{d t}=\alpha R_{0} S \frac{H}{H_{0}}=\frac{d I}{d t}=\alpha R_{0} S\left(1-\frac{I}{H_{o}}\right) \\
& \frac{d L}{d t}=\alpha R_{0} S \frac{H}{H_{0}}-\theta L \\
& \frac{d S}{d t}=\theta L-\alpha S \\
& \frac{d R}{d t}=\alpha S \\
& H_{0}=H+L+S+R=H+I \\
& I=L+S+R
\end{aligned}
$$

where $H_{0}$ is the total amount of susceptible plant tissue assumed to be constant. The parameters $\theta$ and $\alpha$ are the per capita rates of passage from latent to infectious and from infectious to postinfectious, respectively.

The Laplace Transform of the reproduction function for the HLSR model (equation 12) is derived in the Appendix:

$$
\widetilde{\mathfrak{R}}_{H L S R}(\xi)=\frac{R_{0} \alpha \theta}{(\xi+\alpha)(\xi+\theta)}
$$

The rate parameters $\theta$ and $\alpha$ enter equation A12 in a completely symmetrical fashion, so that an interchange of these two parameters does not change the shape of the reproduction curve. For the HLSR model, the mean latent period, $p$, is given by $\theta^{-1}$ and the mean infectious period, $i$, is given by $\alpha^{-1}$. Thus, we can interchange the value of $p$ with the value of $i$ without changing the course of the resulting epidemic. Cunniffe et al. (9) (Table 3) used the prescription that $p$ and $p+i$ must remain constant when comparing the HLSR model to Vanderplank's SP and $\gamma$ distribution model. However, this leads to the ambivalent situation that one HLSR reproduction curve is paired with two different SP models or two different $\Gamma$ models, with the values of $p$ and $i$ interchanged.

Performing the inverse Laplace Transform equation A12 yields two different forms for the reproduction function depending on whether or not $\theta$ and $\alpha$ are equal:

$$
\Re_{H L S R}(\tau, \alpha, \theta)=\left\{\begin{array}{cc}
R_{0} \frac{\alpha^{2} \tau e^{-\alpha \tau}}{\Gamma(2)} & \theta=\alpha \\
R_{0} \frac{\alpha \theta}{\alpha-\theta}\left(e^{-\theta \tau}-e^{-\alpha \tau}\right) & \theta \neq \alpha
\end{array}\right.
$$

Segarra et al. (52) presented only the second form of equation 13, which is not defined for $\theta=\alpha$. Note that the first form in equation $16(\theta=\alpha)$ (Table 2, case 1$)$ is identical to the $\gamma$ distribution (equation 8) with $n=2$ and $\tau_{D}=0$. Furthermore, in the limit that either $\theta$ or $\alpha$ is much larger than the other rate parameter $(\theta>>\alpha$ or $\alpha>>\theta$ ) (Table 2, case 2), the second form in equation 13 ap-

TABLE 2. Laplace Transforms and the first four centered moments for reproduction curves, $\Re(\tau)$, used in the text (equation 5) (33)

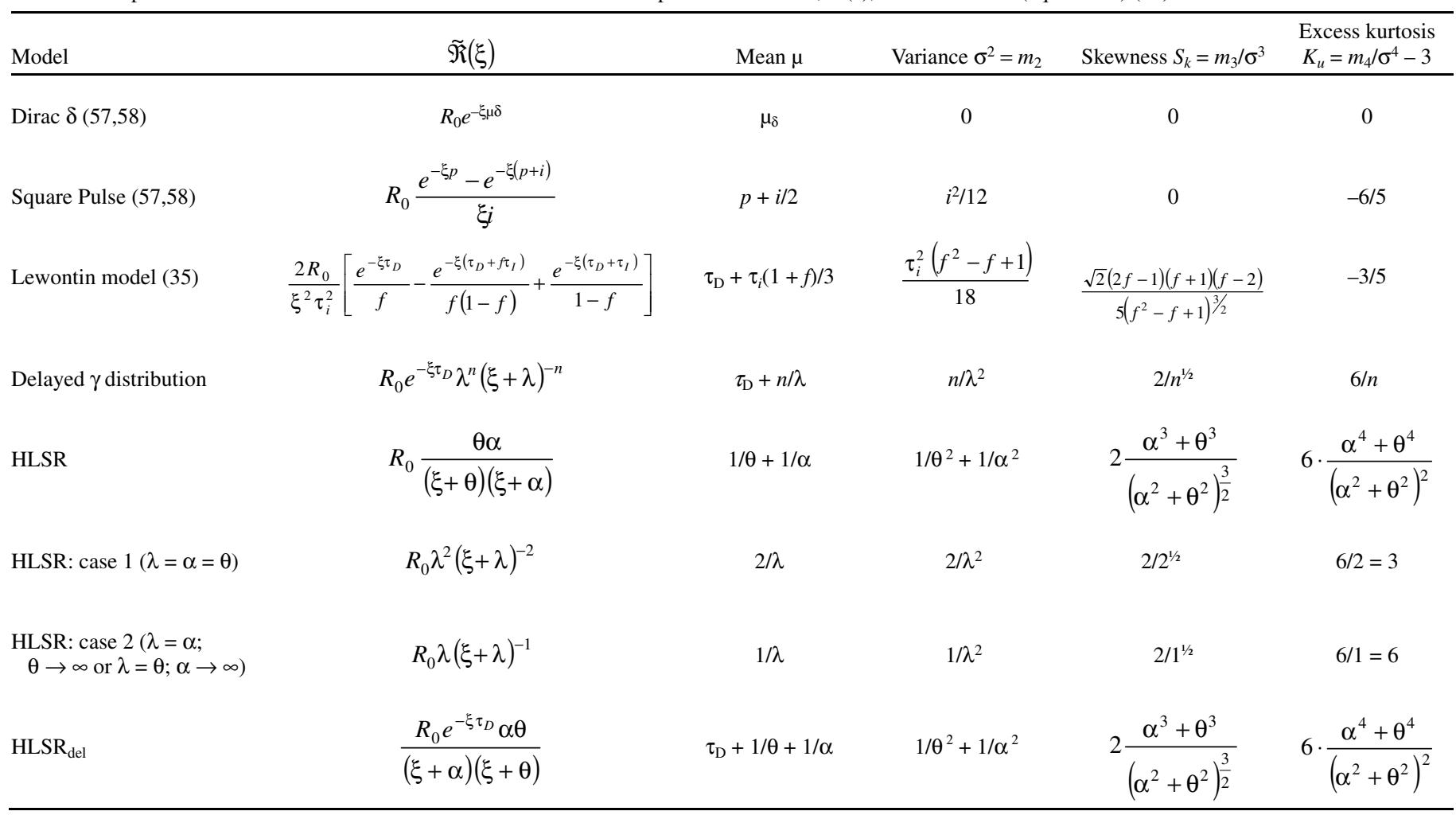


proaches the $\gamma$ distribution (equation 11), with $n=1$ and $\tau_{D}=0$. The moments of $\Re_{H L S R}(\tau)$ are given in Table 2 . In terms of the first two temporal moments ( $\mu$ and $\sigma$ ), equation A12 can be inserted into equation $2 \mathrm{~b}$ to yield:

$$
1=\left.\widetilde{\mathfrak{R}}_{H L S R}(\mu, \sigma)\right|_{\xi=r}=\frac{2 R_{0}}{r^{2} \mu^{2}+2 r \mu-r^{2} \sigma^{2}+2}
$$

Unlike the previous three models (equations 9, 12, and 14), equation 14 cannot be expressed as a function of $r \mu$ times a function of $r \sigma$. For this model, the first two temporal moments of the progeny production curve cannot be varied independently, despite the fact that there are two shape parameters $(\alpha$ and $\theta)$. In fact, the $c_{\mathrm{V}}$ has a minimum value of $2^{-1 / 2} \approx 0.707$ for case 1 and a maximum value of unity for case 2 . However, the value of $r$ obtained from equation 14 always lies between the values of $r$ calculated from equation 9 , with $\tau_{D}=0$ and either $n=1$ or $n=2$.

$H L S R$ model with an explicit time delay, $\tau_{D}\left(H L S R_{d e l}\right)$. The limitations in the range of the $c_{\mathrm{V}}$ in the above model (equation 12; $\left.c_{\mathrm{V}} \geq 2^{-1 / 2} \approx 0.707\right)$ restricts its applicability to many real-world pathosystems, which are characterized by less disperse reproduction curves (Table 4) $\left(c_{\mathrm{V}} \leq 0.35\right)$. The simple introduction of an explicit time lag, $\tau_{D}$, between the time of an initial infection and the entry of the resulting lesion into the latent compartment, $L$ (equation 12), remedies this situation. If, in addition, one assumes that the ratio of $\alpha$ to $\theta$ is constant for any one model, then the $\mu$ and $\sigma$ of the resulting reproduction curve can be varied independently and the value of $c_{\mathrm{V}}$ can range from 0 to unity. The details of this model are derived in the Appendix. The net result is that equation 14 is replaced by

$$
1=\left.\widetilde{\mathfrak{R}}_{\text {HLSRdel }}(\xi)\right|_{\xi=r}=R_{0} e^{-r \mu} \frac{e^{r \sigma(\sin \psi+\cos \psi)}}{(r \sigma \sin \psi+1)(r \sigma \cos \psi+1)}
$$

where the angle $\psi=\arctan (\alpha / \theta)$ is assumed constant for a particular model. Note that the term on the far right side of equation
A16 is expressible as a function of $r \mu$ times a function of $r \sigma$. When $\psi=\pi / 4$ radians $\left(45^{\circ}\right), \alpha=\theta$ and equation A16 is equivalent to equation 12 , with $n=2$. As $\psi$ approaches either $\pi / 4\left(90^{\circ}\right)$ or 0 , equation A16 approaches the value of equation 12, with $n=1$.

The initial infection rate, $r$. Epidemics resulting from Vanderplank's $(57,58)$ SP model (equation 6) yield relationships between the value of $R_{0}$, the basic reproduction number, and $c_{\mathrm{V}}$, the $c_{\mathrm{V}}$ of the reproduction curve $\left(c_{\mathrm{V}}=\sigma / \mu\right)$, to the dimensionless form of the initial infection rate, $r^{\prime}$ (the initial infection rate times the mean time of daughter lesion production: $r^{\prime}=r \mu$ ). This is illustrated in Figure 2, where the isopleths of $r^{\prime}$ are plotted on the $R_{0}-c_{\mathrm{V}}$ plane.

Note that each line in Figure 2 crosses the y-axis at the point where $R_{0}=\exp \left(r^{\prime}\right)$. This corresponds to the Dirac $\delta$ form for the reproduction curve (equation 7). Note also that, as $r^{\prime}$ decreases, the curvature of the lines decrease. This behavior suggests that the proper transformation of variables might simplify this graph. The first three models discussed above (equations 6, 9, and 11) and the HLSR $_{\text {del }}$ model (equation A16) can all be put in the following form:

$$
\begin{gathered}
\widetilde{\mathfrak{R}}_{M}=\frac{R_{0} e^{-r \mu}}{\phi_{M}(r \sigma)}=\left(R_{0}\right)_{\text {eff }} e^{-r \mu}=\frac{\widetilde{\mathfrak{R}}_{\delta}(r \mu)}{\phi_{M}(r \sigma)}=1 \\
\text { or } \widetilde{\mathfrak{R}}_{\delta}(r \mu)=\phi_{M}(r \sigma)
\end{gathered}
$$

where the subscripted "M" stands for the model in question; $\left(R_{0}\right)_{\text {eff }}$, the effective $R_{0}$, is the basic reproductive ratio in a Dirac $\delta$ model (equation 10 with $\mu_{\delta}=\mu$ ), which results in the same initial rate of disease increase, $r$, as the model in question; $\widetilde{R}_{\delta}(r \mu)$ is the Laplace Transform of a Dirac $\delta$-type reproduction curve (equation $7)$; and $\phi_{M}(r \sigma)$ is a model-dependent shape factor.

When $\phi_{S P}$ (equation 15) is plotted versus the product $r \sigma$, the

\begin{tabular}{|c|c|c|}
\hline Symbol & Units $^{\mathrm{a}}$ & Definition \\
\hline$C(\tau)$ & $\mathrm{d}^{-1}$ & Stable lesion age distribution corresponding to the steady state solution (equation $2 \mathrm{a}$ ) to the linearized form of the KM model (equation 1 ) \\
\hline$c_{\mathrm{V}}$ & ND & Coefficient of variation of $\Re(\tau)\left(c_{\mathrm{V}}=\sigma / \mu\right)($ Table 2$)$ \\
\hline$H$ & $N_{s t}$ & Total number of healthy susceptible sites \\
\hline$H_{\max }$ & $N_{s t}$ & Total number of susceptible sites \\
\hline$i$ & $\mathrm{~d}$ & Infectious period \\
\hline$I$ & $N_{s t}$ & Total number of infected sites including $L, S$, and $R$ \\
\hline$I_{0}$ & $N_{s t}$ & Initial number of infected sites including $L, S$, and $R$ \\
\hline$K_{\mathrm{u}}$ & ND & Excess kurtosis of $\mathfrak{R}(\tau)$ (Table 2 ) \\
\hline$l(\tau)$ & ND & Probability of lesion survival to age $\tau$ \\
\hline$L$ & $N_{l e}$ & Number of latently infected sites \\
\hline$m(\tau)$ & $d^{-1}$ & Per capita time rate of progeny production \\
\hline$N$ & $N_{s t}$ & Total number of susceptible sites \\
\hline$P$ & $\mathrm{~d}$ & Latent period \\
\hline$r$ & $\mathrm{~d}^{-1}$ & Logarithmic rate parameter for initial increase in disease (Vanderplank's $r_{l}$ ) (58) \\
\hline$R$ & $N_{s t}$ & Total cumulative number of removed sites \\
\hline$R_{0}$ & ND & Basic reproduction ratio (the ratio of the total number of daughter lesions produced per mother lesions when $H=H_{0}$ ) \\
\hline$\left(R_{0}\right)_{e f f}$ & ND & $\begin{array}{l}\text { Basic reproductive ratio in a Dirac } \delta \text { model (equation } 10 \text {, with } \mu \delta=\mu \text { ), which results in the same initial rate of disease increase, } \\
r \text {, as the model in question }\end{array}$ \\
\hline$R_{\mathrm{n}}$ & ND & The $n$ th-order temporal moment of $\Re(\tau)$ about $\tau=0$ (equation 3 ) \\
\hline$S$ & $N_{l e}$ & Number of infective actively sporulating sites \\
\hline$S_{\mathrm{k}}$ & ND & Skewness of $\Re(\tau)$ (Table 2) \\
\hline$\sigma$ & $\mathrm{d}$ & Standard deviation of $\Re(\tau)$ about $\mu$ \\
\hline$\mu$ & $\mathrm{d}$ & First temporal moment of $\Re(\tau)$ (time between successive generations of the pathogen) \\
\hline$\lambda$ & $d^{-1}$ & Rate parameter for the $\gamma$ distribution form of the reproduction curve (equations 8 and 12) \\
\hline$\xi$ & $d^{-1}$ & Frequency variable for Laplace transforms \\
\hline$\Re(\tau)$ & $d^{-1}$ & Number of daughter lesions produced per mother lesion per unit time (including the effects of removal) \\
\hline$\tau_{\mathrm{D}}$ & $\mathrm{d}$ & Delay time \\
\hline$\tau_{\mathrm{i}}$ & $\mathrm{d}$ & Infectious period for Lewontin model (35) \\
\hline$\tau$ & $\mathrm{d}$ & Age of a lesion \\
\hline$\phi$ & ND & Function of the product $r \sigma$ equal to the ratio: $R_{0} /\left(R_{0}\right)_{\text {eff }}$ \\
\hline$\psi$ & ND & Transformation variable for HLSR del $_{\text {model }}$ \\
\hline
\end{tabular}
curves of Figure 2 become collinear (Fig. 3). This is a result of the nature of the form of equation 6 , which is expressible as a

TABLE 3. Symbols

a $\mathrm{ND}=$ no dimension, $N_{s t}=$ number of sites, $N_{l e}=$ number of lesions, and $\mathrm{d}=$ time (days). 
function of $r \mu$ times a function of $r \sigma$, and the definition the function $\phi$ (equation 15). The value of $\phi_{M}(0)$ is always equal to unity, the derivative of $\phi_{M}$ evaluated at $r \sigma=0$ is always zero, and the function $\phi_{M}$ is monotonically decreasing as the product $r \sigma$ increases. Thus, the effective value of the basic reproductive ratio $\left[\left(R_{0}\right)_{e f f}\right]$ is always larger than $R_{0}$. In similar fashion, all the rest of the models discussed above are plotted in Figure 4 and folded in Figure 5. Note that all of the above theoretical models yield very similar values for $\phi_{M}(r \sigma)$ when the value of $r \sigma<0.5$. In this regime, the resulting epidemic is relatively slow and it takes many generations of the pathogen to infect a major portion of the host. With each succeeding generation of the pathogen, the reproduction curve is convoluted with itself (Appendix). The net result is that, after multiple generations, the flush of new infecteds approaches a normal distribution due to the central limit theorem
(38), regardless of the shape of the original reproductive curve (Fig. 6).

\section{EXPERIMENTAL SPORULATION CURVES}

A collection of experimental sporulation curves obtained from the literature $(5,11,24,29,44,46,48,53)$ is listed in Table 4. Although there is considerable variation among the observed time scales for the various host-pathogen systems, the value of $c_{\mathrm{V}}$ is always $\leq 0.35$. Rather than fitting the aforementioned experimental reproduction curves to one of the above theoretical models in order to estimate the initial infection rate, I suggest the following, more direct approach.

A fourth-order expansion of $\boldsymbol{r}$ in terms of $\ln \left(\boldsymbol{R}_{\mathbf{0}}\right)$. Dublin and Lotka (14) have shown that the natural logarithm of $R_{0}$ can be

TABLE 4. Temporal moments of experimental sporulation curves found in the literature ${ }^{\mathrm{a}}$

\begin{tabular}{|c|c|c|c|c|c|c|}
\hline Pathogen & Temperature $\left({ }^{\circ} \mathrm{C}\right)$ & $\mu$ (days) & $\sigma$ (days) & $c_{\mathrm{V}}$ & $S_{\mathrm{k}}$ & $K_{\mathrm{u}}$ \\
\hline \multirow[t]{4}{*}{ Pseudoperonospora cubensis (cucumber downy mildew) $(11)^{\mathrm{b}}$} & $10-15$ & 10.6 & 1.84 & 0.17 & -0.34 & -0.48 \\
\hline & $10-20$ & 8.5 & 2.07 & 0.24 & -0.17 & -0.63 \\
\hline & $20-25$ & 6.0 & 1.50 & 0.25 & 0.30 & -0.19 \\
\hline & $20-30$ & 5.5 & 0.91 & 0.17 & 0.47 & 0.07 \\
\hline \multirow[t]{3}{*}{ Puccinia striiformis (wheat stripe rust) (43) (Fig. 6, 'Maris Beacon') (50) ${ }^{\mathrm{b}}$} & 10 & 21.5 & 2.9 & 0.14 & 0.02 & -0.70 \\
\hline & 15 & 15.8 & 2.3 & 0.15 & 0.31 & -0.48 \\
\hline & 20 & 13.0 & 1.8 & 0.14 & 0.38 & -0.50 \\
\hline Puccinia hordei (barley leaf rust) $(53)^{\mathrm{c}}$ & 25 & 8.7 & 2.3 & 0.26 & 0.56 & -0.60 \\
\hline Puccinia coronata (oat crown rust) $(24)^{\mathrm{c}}$ & $21-26$ & 15.3 & 3.6 & 0.23 & 0.36 & -0.60 \\
\hline Puccinia recondita (wheat leaf rust) (46) ${ }^{\mathrm{c}}$ & 25 & 17.7 & 6.3 & 0.35 & 0.47 & -0.60 \\
\hline Puccinia graminis (wheat stem rust) (47) ${ }^{\mathrm{c}}$ & 25 & 18.3 & 5.1 & 0.28 & 0.37 & -0.60 \\
\hline Pyricularia oryzae (rice blast) $(30)^{\mathrm{c}}$ & 25 & 12.7 & 4.4 & 0.35 & 0.53 & -0.60 \\
\hline Sphaerotheca fuliginea (cucumber powdery mildew) $(5)^{\mathrm{c}}$ & 25 & 19.0 & 3.9 & 0.21 & 0.15 & -0.60 \\
\hline
\end{tabular}

a Abbreviations: $c_{\mathrm{V}}=$ coefficient of variation, $S_{\mathrm{k}}=$ skewness, and $K_{\mathrm{u}}=$ kurtosis.

$\mathrm{b}$ Moments for these reproduction curves are calculated directly from observation.

${ }^{\mathrm{c}}$ Moments for these reproduction curves are calculated using the Lewontin model (equation 13) as applied by Leonard and Mundt (34).

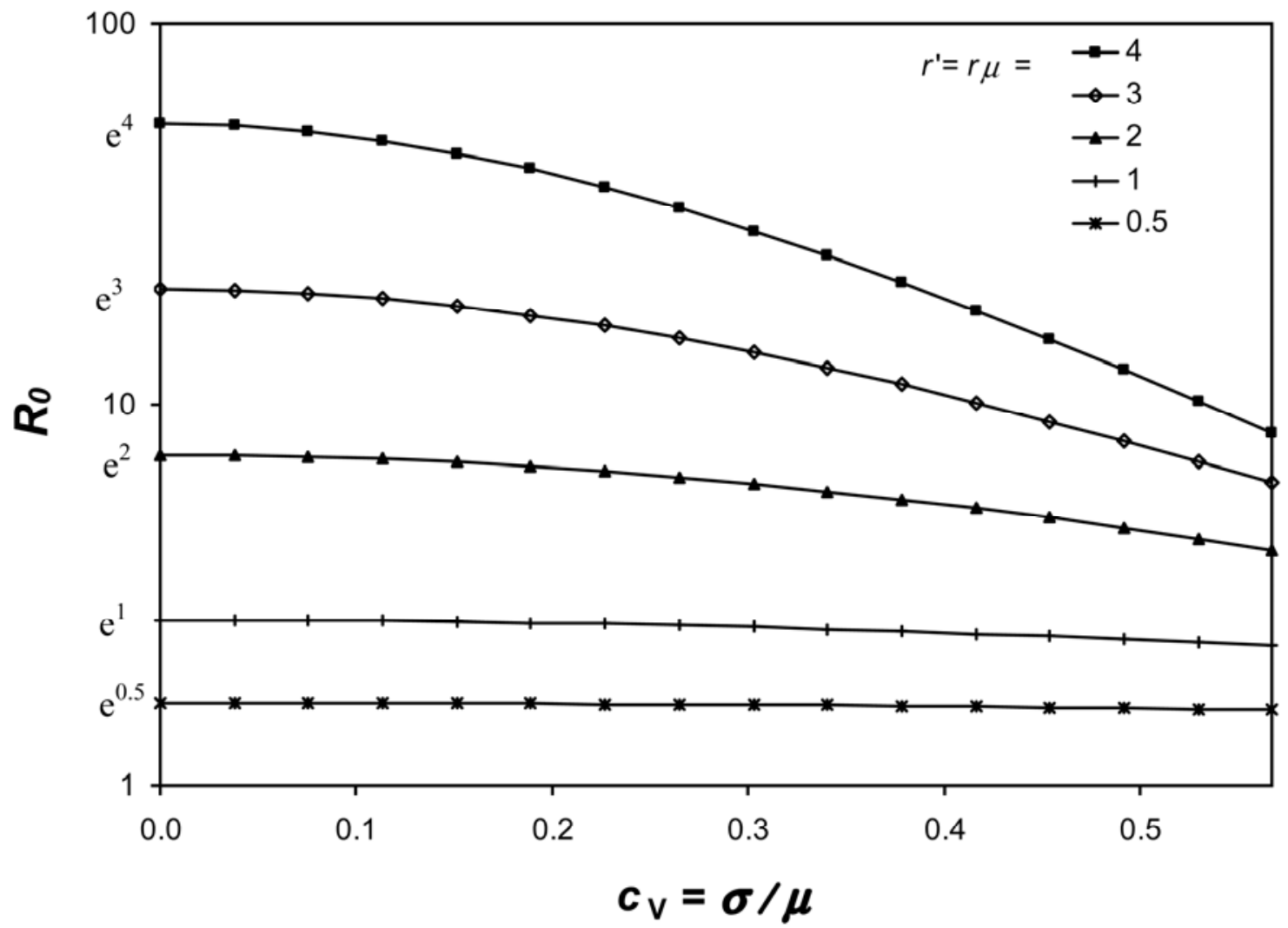

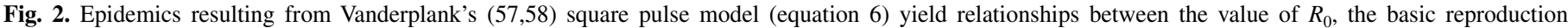

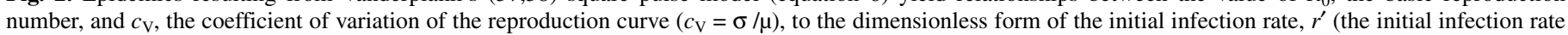
times the mean time of daughter lesion production: $\left.r^{\prime}=r \mu\right)$. Note that each line crosses the $\mathrm{y}$-axis at the point where $R_{0}=\exp \left(r^{\prime}\right)$. 
expressed as the following power series in $r \sigma$ :

$$
\ln \left(R_{0}\right)=\mu r-\frac{1}{2 !} r^{2} \sigma^{2}+\frac{S_{k}}{3 !} r^{3} \sigma^{3}-\frac{K_{u}}{4 !} r^{4} \sigma^{4}-\ldots
$$

This same result was reported by Segarra et al. (52) citing Metz and Diekmann (47), who used the cumulant generating function of the reproduction function, $\Re(\tau)$. Metz and Diekmann (47) also document a quadratic form for $r$ in terms of $R_{0}, \mu$, and $\sigma$, first reported by Dublin and Lotka (14), when the reproductive curve is near normal. Because only the first two cumulants $\left(\mu\right.$ and $\left.\sigma^{2}\right)$ of the normal distribution are nonzero, equation 16 reverts to the following quadratic equation:

$$
\ln \left(R_{0}\right)=\mu r-\frac{1}{2 !} r^{2} \sigma^{2} \text { or } \ln (\phi)=-\frac{r^{2} \sigma^{2}}{2} \Rightarrow \phi=e^{-\frac{r^{2} \sigma^{2}}{2}}
$$

which can be solved for $r$ to yield

$$
r=\frac{1}{\mu c_{V}^{2}}\left(1-\sqrt{1-2 \ln \left(R_{0}\right) c_{V}^{2}}\right)
$$

In the more general case, when the higher-order cumulants are important, the utility of equation 16 is limited, because the series becomes divergent when the quantity $r \sigma$ is greater than unity. Because the characteristic equations (equations 6, 9, 11, and 14) of the various epidemic models described above cannot be explicitly solved for $r$, it would be more useful to have an estimate of $r$ in terms of a power series in $\ln \left(R_{0}\right)$ for more general reproduction functions.

Following Metz and Diekmann (47, pages 153-154), I assume that $r$ can be expressed as a power series in $\ln \left(R_{0}\right)$ :

$$
r=A \ln \left(R_{0}\right)+B \ln \left(R_{0}\right)^{2}+C \ln \left(R_{0}\right)^{3}+D \ln \left(R_{0}\right)^{4}+\cdots
$$

where the coefficients ( $A$ to $D$ ) are evaluated by inserting equation 18 into equation 16 and setting the coefficients of the powers of $\ln \left(R_{0}\right)$ in equation 21 equal to zero, because $r$ must equal 0 when $R_{0}=1$, or, equivalently, when $\ln R_{0}=0$. The resulting fourth-order approximation for $r$ is

$$
r=\frac{\ln \left(R_{0}\right)}{\mu} \cdot\left[\begin{array}{l}
1+\ln \left(R_{0}\right) \frac{c_{V}{ }^{2}}{2}+\left[\ln \left(R_{0}\right)\right]^{2}\left(\frac{c_{V}{ }^{4}}{2}-\frac{S_{k} c_{V}{ }^{3}}{6}\right) \\
{\left[\ln \left(R_{0}\right)\right]^{3}\left(\frac{5 c_{V}{ }^{6}}{8}-\frac{5 S_{k} c_{V}{ }^{5}}{12}+\frac{K_{u} c_{V}{ }^{4}}{24}\right)+\ldots}
\end{array}\right]+
$$

Metz and Diekmann (47, pages 153-154) present only the first two terms of equation 19. This limited result is consistent with second-order approximations for $r$ derived in the literature $(14,47$, $52)$. Because $\ln \left(R_{0}\right)$ can be greater than unity, the convergence of equation 22 critically hinges on the value of the product $\ln \left(R_{0}\right)$. $c_{\mathrm{V}}{ }^{2}$. This condition is not a serious limitation to the applicability of equation 19. Note, an expansion of the square root term in equation 17 in a McClaurin series about $2 \cdot \ln \left(R_{0}\right) c_{\mathrm{V}}{ }^{2}=0$ is identical to equation 19 , with skewness $\left(S_{\mathrm{k}}\right)=$ kurtosis $\left(K_{\mathrm{u}}\right)=0$.

Application of equation 19 to experimental and theoretical reproduction curves. Because the quantities $\mu, c_{\mathrm{V}}, S_{\mathrm{k}}$, and $K_{\mathrm{u}}$ (Table 1) can all be directly estimated from an experimentally obtained reproduction curve, equation 19 can be used to give the initial infection rate, $r$, in terms of the value of the basic reproductive ratio, $R_{0}$. The function $\varphi$ (equation 15) can then be compared with the results obtained from the theoretical model reproduction curves (Fig. 7).

As noted before, the characteristic equations (equations 6, 9, 11 , and 14) of the various epidemic models described above cannot be explicitly solved for $r$. However, equation 19 can be used to express $r$ in terms of $\ln \left(R_{0}\right), \mu, \sigma, S_{\mathrm{k}}$, and $K_{\mathrm{u}}$ using the formulae in Table 2. The resultant value of $r$, thus calculated, can then be inserted into the characteristic equations (equations 6, 9, 11 , and 14) of the various models to test for consistency. For epidemics characterized by values of $r \mu<5$, the value of $r$ was

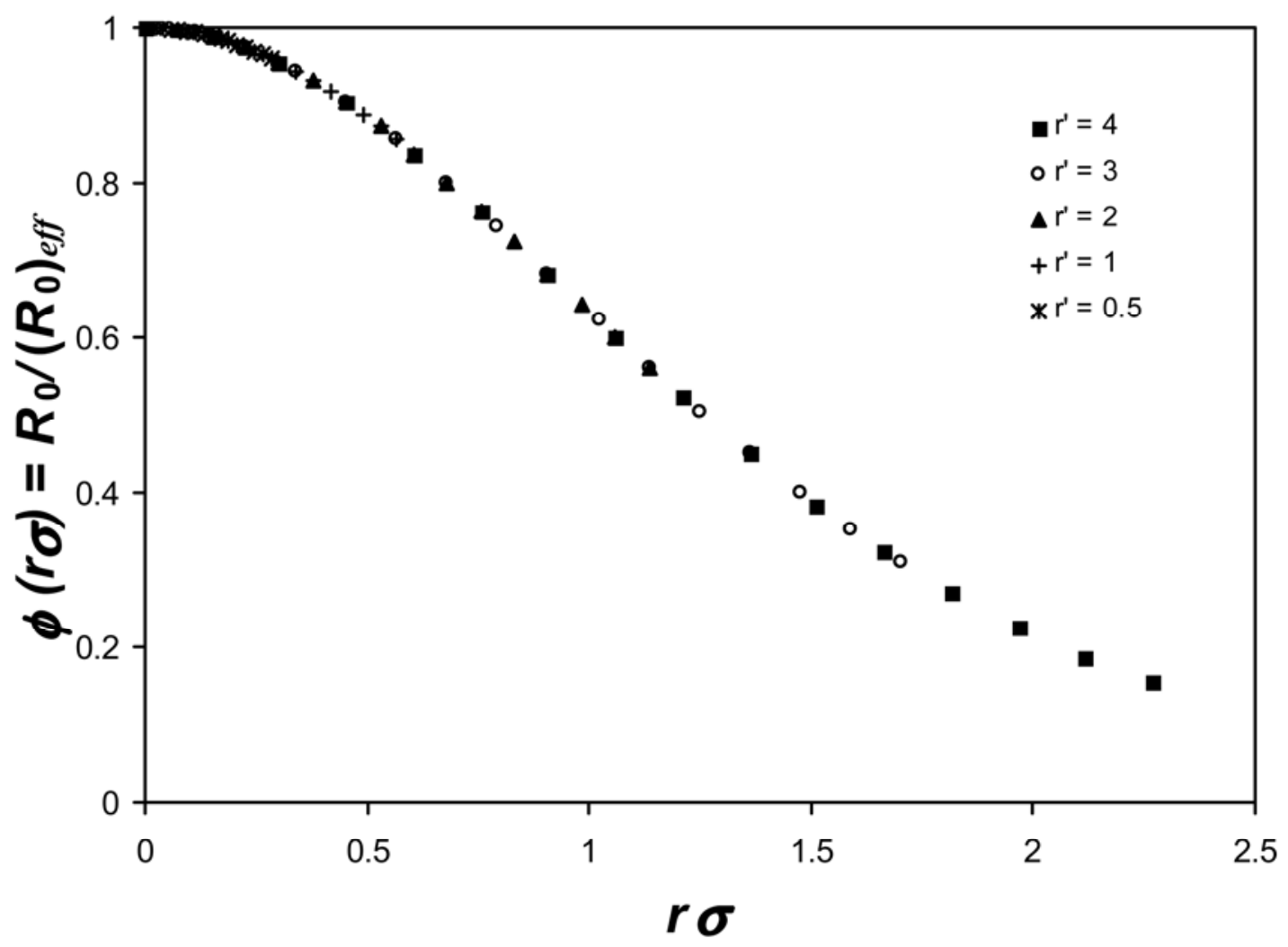

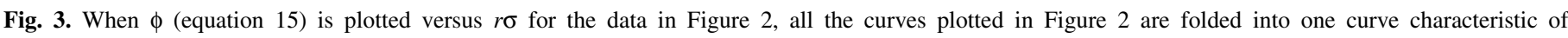
Vanderplank's square pulse model $(57,58)$. 
determined to within $2 \%$ by equation 19 , regardless of the functional form of the reproduction curve. For epidemics characterized by values of $r \mu<3$, the percent error in $r$ never exceeded $0.5 \%$.

\section{DISCUSSION}

In theoretical epidemiology, compartmental models are often used with individuals classified as healthy, $H$; latent, $L$; infectious, $S$; and postinfectious, $R$. Transition from one stage to the next is usually modeled as an exponential decay (equation 12). I have shown that the minimal $c_{\mathrm{V}}$ for such a model is equal to one-half the square root of $2(\approx 0.707)$, which is twice the value obtained from experimental sporulation curves (Table 4). Thus, this model is not suitable to describe epidemics involving these fungal pathogens. However, I have suggested a modification to the above model (equation A16, HLSR del $_{\text {l }}$ ) that includes an explicit delay time between infection and the exponential decay to the infectious state. This change provides the flexibility needed to mimic the observed reproductive behavior of actual fungal pathogens.

An examination of Figure 7 reveals that a wide range of fungal pathosystems fall into a relatively narrow band on a plot of $\phi$ versus $r \sigma$. This band is bounded by the normal curve result (equation $17, S_{\mathrm{k}}=0, K_{\mathrm{u}}=0$ ) and the $\gamma$ distribution model with $n=$ $4\left(S_{\mathrm{k}}=1, K_{\mathrm{u}}=1.5\right)$. This is due to the fact that both the skewness, $S_{\mathrm{k}}$, and the excess kurtosis, $K_{\mathrm{u}}$, of observed sporulation curves have an absolute value $<0.6$ (Table 4). Theoretical models that predict functional forms for the reproductive curve characterized by larger values for these higher-order moments are not applicable to epidemics caused by these pathogens. The major problem is that theoretical curves go on to infinity. The moments of these functions of infinite extent are artificially inflated by small predicted reproduction in an extended temporal tail. For field crops in temperate regions, there is a limited time period $(\approx 100$ to 120 days) over which pathogen reproduction matters. Thus, a more realistic evaluation of a mathematical reproduction curve must involve a truncation of the production of pathogen progeny somewhat before the host crop disappears from the field. A simple fit of a theoretical curve over a finite region of time must not be extrapolated ad absurdum.

In conclusion, I have shown both by analytical methods and by successive approximation that the first two temporal moments of the reproduction curve provide an excellent approximation for the relation between the initial infection rate, $r$, and the basic reproductive ratio, $R_{0}$, for slow epidemics $(r \sigma<0.5)$. However, for faster epidemics, correction terms involving the third- and fourthorder moments $\left(S_{\mathrm{k}}\right.$ and $\left.K_{\mathrm{u}}\right)$ must be included (equation 19) in the relationship.

\section{APPENDIX}

The Laplace Transform methods applied to solution of the linearized KM equation. Assuming $I \ll H_{\max }$ and $H(t) \cong H_{\max }$, we can solve equation 2 by the method of Laplace Transforms. The Laplace Transform $\breve{f}(\xi)$ of a function $f(t)$ is defined by

$$
\tilde{f}(\xi)=\int_{0}^{\infty} \exp \left(-\xi t^{\prime}\right) \cdot f\left(t^{\prime}\right) d t^{\prime}
$$

Thus, the integral in equation $2 b$ is, by definition, the Laplace Transform of $\Re(\tau)$ and equation $2 b$ is simply an expression resulting from the solution of equation 1 using the method of Laplace Transforms. In fact, equation $2 b$ can be simply written as

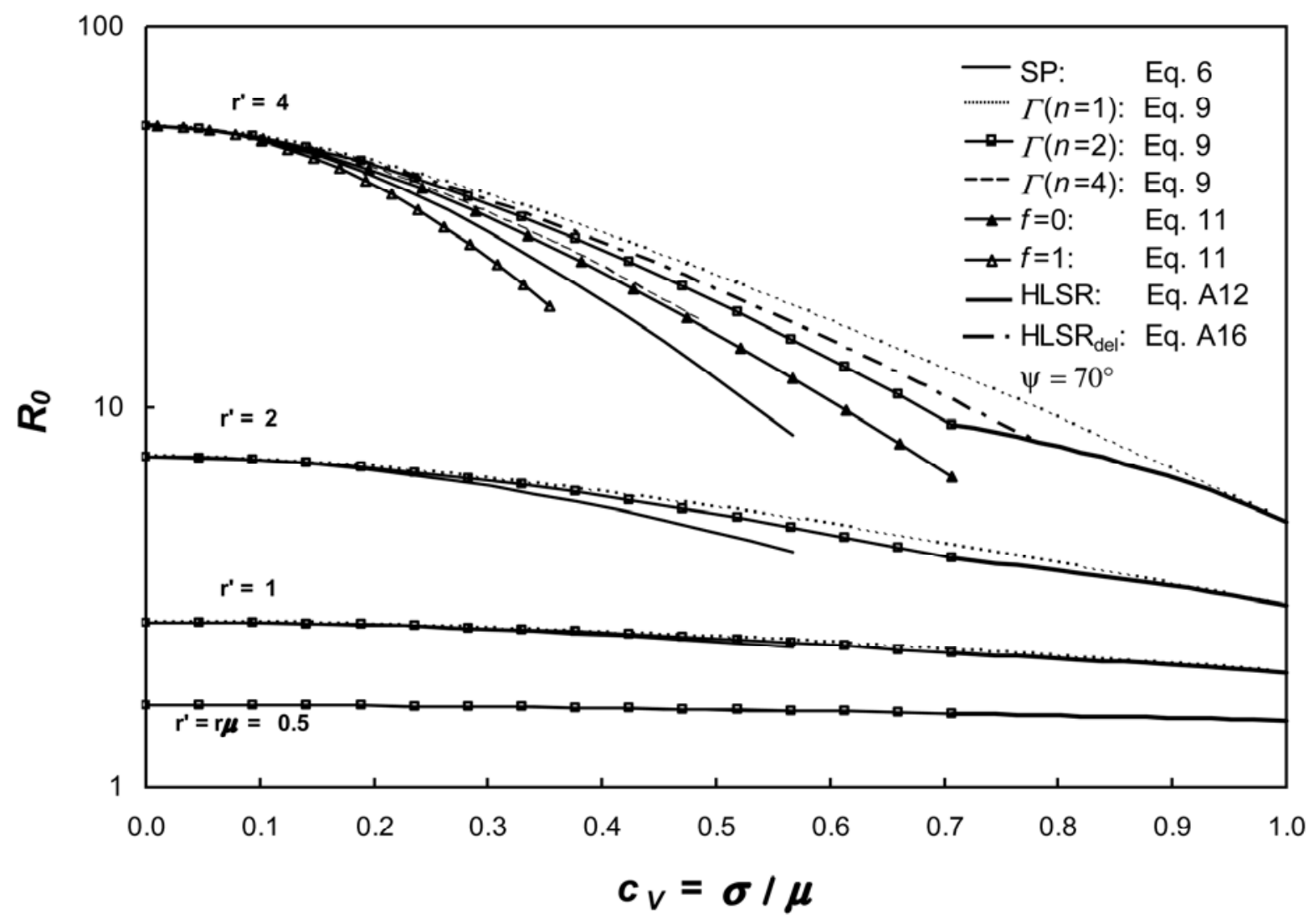

Fig. 4. In analogy with Figure 2, epidemics resulting from all the models discussed in the text yield relationships between the value of $R_{0}$, the basic reproduction number, and $c_{\mathrm{V}}$, the coefficient of variation of the reproduction curve $\left(c_{\mathrm{V}}=\sigma / \mu\right)$, to the dimensionless form of the initial infection rate, $r^{\prime}$ (the initial infection rate times the mean time of daughter lesion production: $r^{\prime}=r \mu$ ). For the sake of clarity, the curves for all eight models are only shown for $r^{\prime}=4$. Note that each line crosses the y-axis at the point where $R_{0}=\exp \left(r^{\prime}\right)$, except for the HLSR (equation A12) model, which has a minimum value for $c_{\mathrm{V}}$ of $2^{-1 / 2}(\approx 0.707)$. The HLSR $\mathrm{del}$ (equation A16) model is bounded by the $\Gamma 1$ and $\Gamma 2$ curves above. 
$\widetilde{R}(r)=1$ (47, page 145). Denoting a time derivative with an overdot and multiplying equation 12 by $\exp \left(-\xi^{\prime}\right)$ and integrating from $t^{\prime}=0$ to $\infty$, one obtains:

$$
\begin{gathered}
\tilde{I}(\xi)=\tilde{I}(\xi) \cdot \widetilde{\mathfrak{R}}(\xi)+\tilde{g}(\xi) \\
\tilde{I}(\xi)=\frac{\tilde{g}(\xi)}{1-\widetilde{\mathfrak{R}}(\xi)}
\end{gathered}
$$

where use has been made of the convolution theorem for Laplace Transforms (i.e., the Laplace Transform of the convolution integral of two functions is equal to the product of the Laplace Transforms of the two functions) (33). In addition, assuming that the function $g(t)$ is a spike function at $t=0$ with area $I_{0}[g(t)=$ $I_{0} \delta(t), \delta(t)$ is the Dirac $\delta$ function] (13), then $\tilde{g}(\xi)=I_{0}$.

The above expression (equation $\mathrm{A} 2 \mathrm{~b}$ ) can be used to generate solutions to the linearized form of equation 1 . These solutions are exponential functions of time whose coefficients are the roots of the denominator in equation $\mathrm{A} 2 \mathrm{~b}$ (33). In complex number theory the number 1 can be expressed as $1=\exp (2 \pi n j)$, where $n$ is any positive or negative integer $(n=\ldots,-2,-1,0,1,2, \ldots)$ and $j$ is the square root of -1 . Substituting these values into the left side of equation $2 \mathrm{~b}$ leads to one real root, $r$, (for $n=0$ ) and an infinite number of complex conjugate pairs of roots, which yield transitory oscillating components that decay after a few generations (41). Thus equation $2 \mathrm{~b}$ can be written as

$$
1=\int_{0}^{\infty} \exp \left(-r t^{\prime}\right) \cdot \Re\left(t^{\prime}\right) d t^{\prime}=\left.\widetilde{\mathfrak{R}}(\xi)\right|_{\xi=r}
$$

and the Laplace Transform of the reproduction function evaluated at $\xi=r$ has a value of unity (47, page 145 ). Note that Laplace
Transform of the reproduction function evaluated at $\xi=0$ is equal to $R_{0}$ by definition (33).

The epidemic as a series of pulses. One can gain some insight into the nature of $\phi_{M}(r \sigma)$, the model-dependent shape factor, by reexamining the Laplace Transformed solution of the linearized $\mathrm{KM}$ equation (equation $\mathrm{A} 2 \mathrm{~b}$ ).

$$
\tilde{I}(\xi)=\frac{I_{0}}{1-\widetilde{\mathfrak{R}}_{M}(\xi)}
$$

where, once again, the subscripted " $\mathrm{M}$ " stands for the model in question and it has been assumed that $\tilde{g}(\xi)=I_{0}$. The above expression can be expanded into the following power series by performing the division:

$$
\begin{aligned}
& \tilde{I}(\xi)=I_{0} \cdot\left(1+\widetilde{\mathfrak{R}}_{M}(\xi)+\widetilde{\mathfrak{R}}_{M}^{2}(\xi)+\widetilde{\mathfrak{R}}_{M}^{3}(\xi)+\cdots\right) \\
& \text { or since } \\
& \widetilde{\mathfrak{R}}_{M}(\xi)=R_{0} e^{-\xi \mu} \phi_{M}^{-1}(\xi \sigma) \text { (by Eq. 18) } \\
& \text { one obtains } \\
& \tilde{I}(\xi)=I_{0} \cdot \sum_{k=0}^{\infty} R_{0}^{k} e^{-k \xi \mu} \phi_{M}^{-k}(\xi \sigma)
\end{aligned}
$$

The summation in the above equation represents a series of pulses of daughter lesion production, the $k$ th pulse, $\Delta I_{k}$, defined by

$$
\Delta \tilde{I}_{k}(t)=\frac{I_{0} R_{0}^{k} e^{-k \xi \mu}}{\widetilde{\phi}_{M}^{k}(\xi \sigma)}
$$

Each successive pulse is delayed by a generation time, $\mu$, when compared with preceding pulse. The first pulse $(k=0)$ is a spike

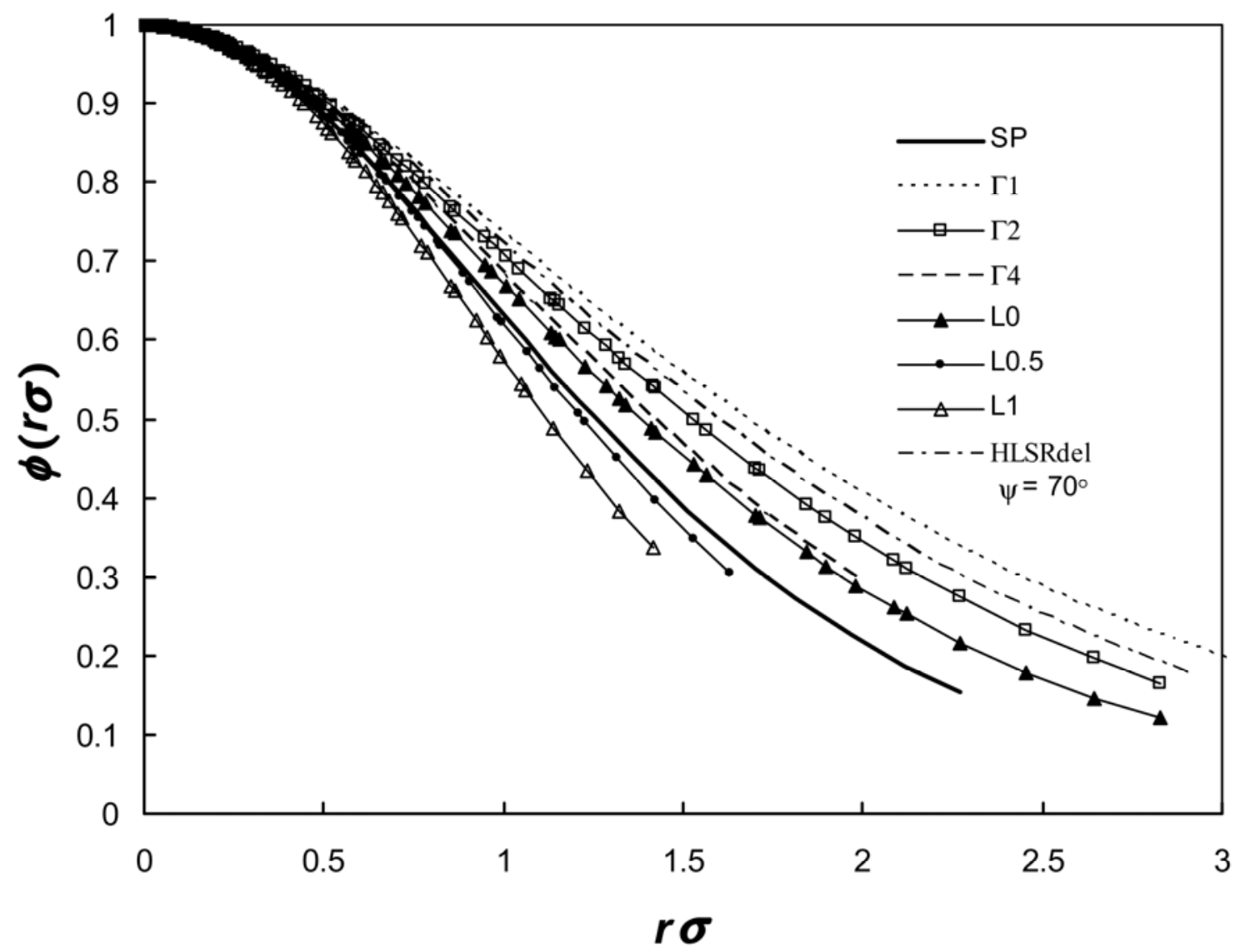

Fig. 5. When $\phi$ (equation 15) is plotted versus $r \sigma$ for the data in Figure 4, each set of curves plotted in Figure 4 (equations 6, 9, and 11), except for the HLSR model (equation A12), are folded into one curve characteristic of the reproduction curve. The HLSR del (equation A16) model is bounded by the $\Gamma 1$ and $\Gamma 2$ curves above. 
function at the origin, $t=0$, and has an area equal to $I_{0}$. The $k$ th pulse has an area $R_{0}$ times the area of the $(k-1)$ th pulse. This process is illustrated in Figure 6. In general, the mean time of daughter lesion production of $\Delta I_{k}$ is given by $\mu_{k}=k \cdot \mu_{M}$, and the variance is $k \cdot \sigma_{M}{ }^{2}$. Due to the central limit theorem (38), $\Delta I_{k}$ approaches a normal distribution as $k$ gets large, regardless of the shape of initial reproduction curve. In addition, this limiting Normal distribution is identical for all models when the firstgeneration mean and variance are the same.

Lewontin's Model. The Laplace Transform for the triangular reproduction curve (equation 10) suggested by Lewontin (35) is

$$
1=\left.\widetilde{\Re}_{L}(\xi)\right|_{\xi=r}=\frac{2 R_{0}}{r^{2} \tau_{i}^{2} f(1-f)}\left[\begin{array}{l}
(1-f)\left(e^{-r \tau_{D}}-e^{-r\left(\tau_{D}+f \tau_{i}\right)}\right)- \\
f\left(e^{-r\left(\tau_{D}+f \tau_{i}\right)}-e^{-r\left(\tau_{D}+\tau_{i}\right)}\right)
\end{array}\right]
$$

The first two temporal moments for this function are given by

$$
\begin{gathered}
\mu=\theta=\tau_{D}+\tau_{i}(1+f) / 3 \\
\sigma^{2}=\frac{\tau_{i}^{2}\left(f^{2}-f+1\right)}{18}
\end{gathered}
$$

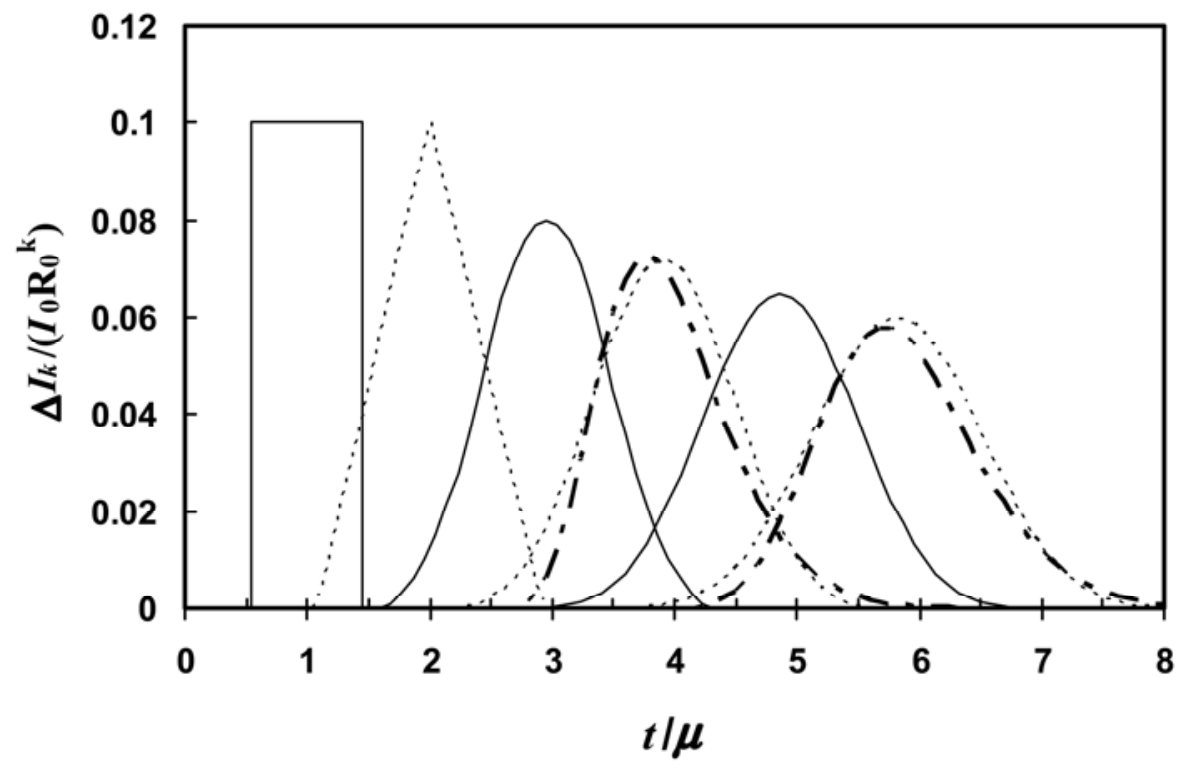

Fig. 6. Alternate solid- and dashed-line figures represent the sequential generations of daughter lesion production for a Vanderplank square pulse reproduction model (equation 6). For comparison, the fourth and sixth generations are shown for a delayed $\gamma$ function model of order 2 (equation $8, n=2$; bold dot-dash lines). Curves illustrated are normalized, so that the area under each curve is unity. Both reproduction curves have the same mean, $\mu$, and variance, $\sigma^{2}$, and both approach

\begin{tabular}{|c|c|c|}
\hline Pathogen & Temperature $\left({ }^{\circ} \mathrm{C}\right)$ & Label \\
\hline \multirow{4}{*}{$\begin{array}{l}\text { Pseudoperonospora cubensis } \\
\text { (cucumber downy mildew, 11) }\end{array}$} & $10-15$ & PsC1015 \\
\hline & $10-20$ & PsC1020 \\
\hline & $20-25$ & $\mathrm{PsC2025}$ \\
\hline & $20-30$ & $\mathrm{PsC} 2030$ \\
\hline \multirow{3}{*}{$\begin{array}{l}\text { Puccinia striiformis }{ }^{\text {}} \text { (wheat stripe rust, } \\
\text { 43: Fig. } 6 \text { cultivar "Maris Beacon", 50) }\end{array}$} & 10 & PS10 \\
\hline & 15 & PS15 \\
\hline & 20 & PS20 \\
\hline $\begin{array}{l}\text { Puccinia hordei }{ }^{\mathrm{b}} \\
\text { (barley leaf rust, 53) }\end{array}$ & 25 & $\mathrm{PH} 25$ \\
\hline $\begin{array}{l}\text { P. coronata }{ }^{b} \\
\text { (oat crown rust, 24) }\end{array}$ & $21-26$ & PC2126 \\
\hline $\begin{array}{l}\text { P. recondita }{ }^{b} \\
\text { (wheat leaf rust, 46) }\end{array}$ & 25 & PR25 \\
\hline $\begin{array}{c}\text { P. graminis }{ }^{b} \\
\text { (wheat stem rust, 47) }\end{array}$ & 25 & PG25 \\
\hline $\begin{array}{l}\text { Pyricularia oryzae } \\
\text { (rice blast, } 9,30 \text { ) }\end{array}$ & 25 & PO25 \\
\hline $\begin{array}{l}\text { Sphaerotheca fuliginea } \\
\text { (cucumber powdery mildew, } 5 \text { ) }\end{array}$ & 25 & SF25 \\
\hline
\end{tabular}
the same normal distribution as $k$, the number of generations, gets larger.

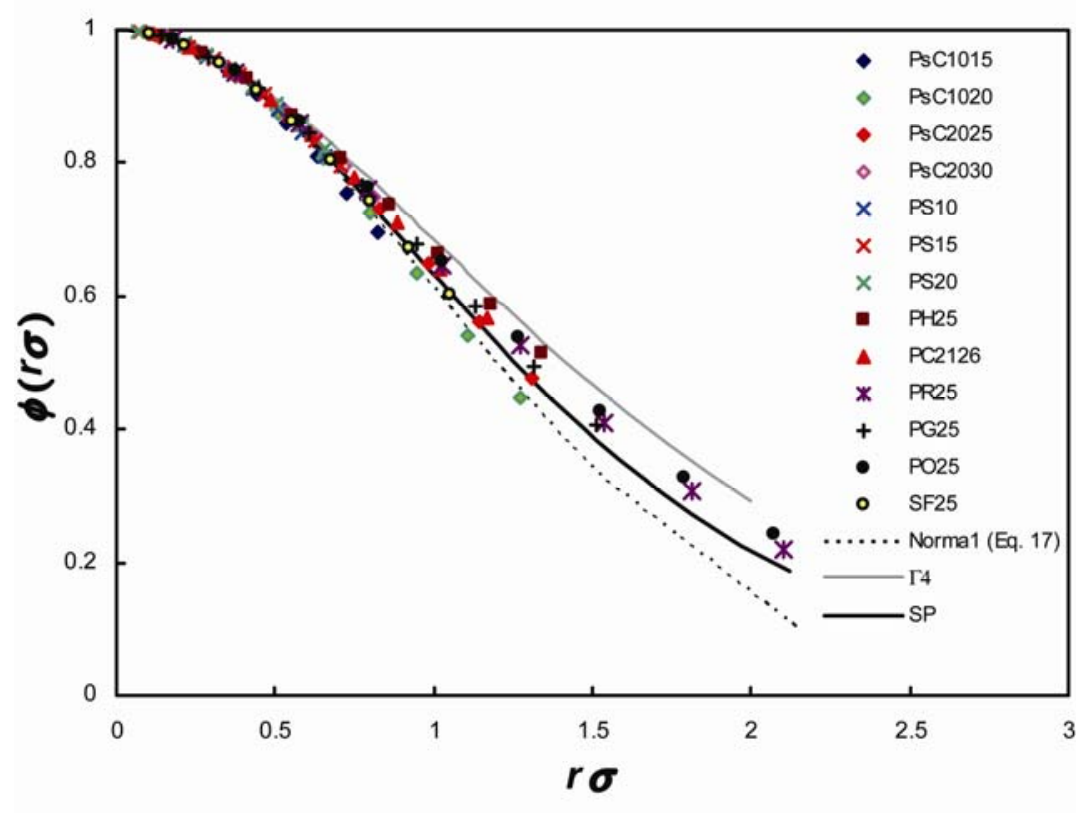

Fig. 7. Equation $15(\varphi)$ is plotted versus the product $r \sigma$ for the experimental reproduction curves shown in Tables 4 and 5 . Plotted points represent a range of the basic reproduction ratio, $R_{0}$, from 1.5 to 150 . For comparison, the plots of $\varphi$ for the square pulse, $\Gamma 4$, and the Normal Distribution (equation 17) are also shown. 
Solving equations A4 and A5 for $\tau_{D}, \tau_{i}, \tau_{D}+f \tau_{i}$, and $\tau_{D}+\tau_{i}$ yields

$$
\begin{aligned}
& \tau_{i}=\sigma \frac{3 \sqrt{2}}{\sqrt{1-f+f^{2}}} \\
& \tau_{D}=\mu-\sigma \frac{\sqrt{2}(1+f)}{\sqrt{1-f+f^{2}}} \\
& \tau_{D}+f \tau_{i}=\mu+\sigma \frac{\sqrt{2}(2 f-1)}{\sqrt{1-f+f^{2}}} \\
& \tau_{D}+\tau_{i}=\mu+\sigma \frac{\sqrt{2}(2-f)}{\sqrt{1-f+f^{2}}}
\end{aligned}
$$

Inserting equation A6 into equation 11 gives

$$
\widetilde{\mathfrak{R}}_{L}(r)=R_{0} e^{-r \mu} \frac{F}{9 r^{2} \sigma^{2} f(1-f)}\left[\begin{array}{l}
(1-f) e^{\left(r \sigma \frac{\sqrt{2}(1+f)}{\sqrt{F}}\right)}-e^{\left(-r \sigma \frac{\sqrt{2}(2 f-1)}{\sqrt{F}}\right)} \\
f e^{\left(-r \sigma \frac{\sqrt{2}(2-f)}{\sqrt{F}}\right)}
\end{array}\right]
$$

$$
\text { where } F=\left(1-f+f^{2}\right)
$$

The first two terms in expression A7 constitute the transform of the Dirac $\delta$ reproduction curve (equation 7). The remainder of equation $\mathrm{A} 7$ is a function of the product $r \sigma$. If the exponentials within the parenthetical expression in equation A7 are expanded in a Taylor series, the first two terms and the fourth term cancel to zero and the quadratic term is the inverse of the fraction outside the parenthesis, so that equation A7 can be expressed as

$$
R_{0} e^{-\mathrm{r} \mu} \cdot\left[1+\left(r^{2} \sigma^{2}\right)+\text { H.O.T. }\right]
$$

where H.O.T. signifies higher-order terms. Thus, the right-hand side of equation 11 (equation A7) approaches the Dirac $\delta$ value (i.e., $R_{0} e^{-\mathrm{ru}}$; equation 7$)$ as $\sigma^{2}$ goes to zero.

Compartmental model (HLSR). For the sake of convenience, equation 12 in the text is repeated here $(10,26,49)$ :

$$
\begin{aligned}
& H_{\max }=H+L+S+R \\
& I=L+S+R \\
& -\frac{d H}{d t}=R_{0} \alpha S \frac{H}{H_{\max }}=\frac{d I}{d t}=R_{0} \alpha S\left(1-\frac{I}{H_{\max }}\right) \\
& \frac{d L}{d t}=R_{0} \alpha S\left(1-\frac{I}{H_{\max }}\right)-\theta L \\
& \frac{d S}{d t}=\theta L-\alpha S \\
& \frac{d R}{d t}=\alpha S
\end{aligned}
$$

Assuming $H_{\max }$ is a constant (16-19) and $I<<H_{\max }$, then equation 12 is linear. Taking the Laplace Transform of equation 12 and using the general form of the Laplace Transform of a derivative (33) yields

$$
\begin{aligned}
& \xi \tilde{I}-I(0)=R_{0} \alpha \tilde{S} \\
& \xi \tilde{L}-L(0)=R_{0} \alpha \tilde{S}-\theta \tilde{L} \\
& \xi \tilde{S}-S(0)=\theta \tilde{L}-\alpha \tilde{S} \\
& \xi \tilde{R}-R(0)=\alpha \tilde{S}
\end{aligned}
$$

The transform of the reproduction curve for this model (HLSR) can be obtained by setting $L(0)=1$ and $S(0)=R(0)=0$ and assuming that no further infection ensues $(13,52)$. Thus we have the following two equations:

$$
\begin{aligned}
& \xi \tilde{L}-1=-\theta \tilde{L} \rightarrow \tilde{L}=\frac{1}{\xi+\theta} \\
& \xi \tilde{S}=\theta \tilde{L}-\alpha \tilde{S} \rightarrow \tilde{S}=\frac{\theta \tilde{L}}{\xi+\alpha}
\end{aligned}
$$

Which can be combined to yield

$$
\tilde{S}=\frac{\theta}{(\xi+\alpha)(\xi+\theta)}
$$

from which we can obtain the Laplace Transform of the reproduction function:

$$
\widetilde{\mathfrak{R}}_{H L S R}(\xi)=\frac{R_{0} \alpha \theta}{(\xi+\alpha)(\xi+\theta)}
$$

because $R_{0} \alpha$ is the number of daughter lesions produced per unit time per sporulating mother lesion.

Compartmental model with an explicit delay $\left(\operatorname{HLSR}_{\text {del }}\right)$. If one introduces an explicit time delay, $\tau_{D}$, before a new lesion enters the $L$ compartment in the HLSR model, equation 13 becomes

$$
\Re_{H L S R d e l}\left(\tau, \alpha, \theta, \tau_{D}\right)=\left\{\begin{array}{cc}
0 & \tau<\tau_{D} \\
R_{0} \frac{\alpha^{2}\left(\tau-\tau_{D}\right) e^{-\alpha\left(\tau-\tau_{D}\right)}}{\Gamma(2)} & \tau \geq \tau_{D} ; \theta=\alpha \\
R_{0} \frac{\alpha \theta}{\alpha-\theta}\left(e^{-\theta\left(\tau-\tau_{D}\right)}-e^{-\alpha\left(\tau-\tau_{D}\right)}\right) & \tau \geq \tau_{D} ; \theta \neq \alpha
\end{array}\right.
$$

and equation A12 becomes

$$
\widetilde{\mathfrak{R}}_{\text {HLSRdel }}(\xi)=\frac{R_{0} e^{-\xi \tau_{D}} \alpha \theta}{(\xi+\alpha)(\xi+\theta)}
$$

The first two temporal moments of equation A13 are given by

$$
\begin{aligned}
& \mu=\tau_{D}+\frac{1}{\alpha}+\frac{1}{\theta} \\
& \sigma^{2}=\frac{1}{\alpha^{2}}+\frac{1}{\theta^{2}}
\end{aligned}
$$

Introducing a change of variables such that

$$
\begin{aligned}
& \psi=\tan ^{-1}\left(\frac{\alpha}{\theta}\right), \\
& \frac{1}{\alpha}=\sigma \cos \psi, \text { and } \frac{1}{\theta}=\sigma \sin \psi
\end{aligned}
$$

and inserting equation A14 into equation $2 \mathrm{~b}$ yields

$$
1=\left.\widetilde{\mathfrak{R}}_{\text {HLSRdel }}(\xi)\right|_{\xi=r}=R_{0} e^{-r \mu} \frac{e^{r \sigma(\sin \psi+\cos \psi)}}{(r \sigma \sin \psi+1)(r \sigma \cos \psi+1)}
$$

Note that the term on the far right-hand side of equation A16 is expressible as a function of $r \mu$ times a function of $r \sigma$. When fitting equation A13 to an experimental reproduction curve with measured $\mu$ and $\sigma$, the delay time, $\tau_{D}$, is given by

$$
\tau_{D}=\mu-\sigma(\sin \psi+\cos \psi)=\mu-\sqrt{2} \sigma \cos (\psi-\pi / 4)
$$

\section{ACKNOWLEDGMENTS}

I thank an anonymous reviewer for helpful suggestions that improved both the readability and substance of this article. 


\section{LITERATURE CITED}

1. Anderson, R. M., and May, R. M. 1979. Population biology of infectious diseases: Part 1. Nature 280:361-367.

2. Anderson, R. M., and May, R. M. 1979. Population biology of infectious diseases: Part 2. Nature 280:455-461.

3. Anderson, R. M., and May, R. M. 1986. The invasion, persistence, and spread of infectious diseases within animal and plant communities. Phil. Trans. R. Soc. Lond. B314:533-570.

4. Aylor, D. E. 1998. The aerobiology of apple scab. Plant Dis. 82:838-849.

5. Bashi, E., and Aust, H. J. 1980. Quality of spores produced in cucumber powdery mildew compensate for their quantity. Z. Pflanzenkrankh. Pflanzenschutz 87:594-599.

6. Berger, R. D. 1975. Disease incidence and infection rates of Cercospora apii in plant spacing plots. Phytopathology 65:485-487.

7. Berger, R. D., and Jones, J. W. 1985. A general model for disease progress with functions for variable latency and lesion expansion on growing host plants. Phytopathology 75:792-797.

8. Burdon, J. J., and Chilvers, G. A. 1976. Controlled environment experiments on epidemics of barley mildew in different density host stands. Oecologia 26:61-72.

9. Cunniffe, N. J., Stutt, R. O. J. H., van den Bosch, F., and Gilligan, C. A. 2012. Time-dependent infectivity and flexible latent and infectious periods in compartmental models of plant disease. Phytopathology 102:365-380

10. Chan, M. S., and Jeger, M. J. 1994. An analytical model of plant virus disease dynamics with roguing and replanting. J. Appl. Ecol. 31:413-427.

11. Cohen, Y., and Rotem, J. 1971. Rate of lesion development in relation to sporulating potential of Pseudoperonospora cubensis in cucumber. Phytopathology 61:265-268.

12. Diekman, O., Heesterbeek, J. A. P., and Metz J. A. J. 1990. On the definition and the computation of the basic reproduction ratio $R_{0}$ in models for infectious diseases in heterogeneous populations. J. Math. Biol. 28:365-382.

13. Dirac, Paul, 1958. Page 58 in: Principles of Quantum Mechanics, 4th ed. Oxford at the Clarendon Press, Oxford, UK.

14. Dublin, L. I., and Lotka, A. J. 1925. On the True Rate of Natural Increase. J. Am. Stat. Assoc. 20:151:305-339.

15. Feller, W. 1941. On the integral equation of renewal. Ann. Math. Stat. 12:243-267.

16. Ferrandino, F. J. 1989. Spatial and temporal variation of a defoliating plant disease and reduction in yield. Agric. For. Meteorol. 47:273-290.

17. Ferrandino, F. J. 2008. The effect of crop growth and canopy filtration on the dynamics of plant disease epidemics spread by aerially dispersed spores. Phytopathology 98:492-503.

18. Ferrandino, F. J. 2012. Time scales of inoculum production and the dynamics of the epidemic. Phytopathology 102:728-732.

19. Ferrandino, F. J., and Elmer, W. H. 1992. Reduction in tomato yield due to Septoria leaf spot. Plant Dis. 76:208-211.

20. Ficke, A., Gadoury, D. M., and Seem, R. C. 2002. Ontogenic resistance and plant disease management: A case study of grape powdery mildew. Phytopathology 92:671-675.

21. Getz, W. M., and Lloyd-Smith, W. M. 2005. Basic Methods for Modeling the Invasion and Spread of Contagious Diseases. DIMACS Series in Discrete Mathematics and Theoretical Computer Science. American Mathematical Society, Rutgers University.

22. Goudriaan, J., and Monteith, J. L. 1990. A mathematical function for crop growth based on light interception and leaf area expansion. Ann. Bot. 66:695-701.

23. Goudriaan, J., and Van Laar, H. H. 1994. Modelling Potential Crop Growth Processes. Springer Publishing Co., New York.

24. Heagle A. S., and Moore, M. B. 1970. Some effects of moderate adult plant resistance to crown rust of oats. Phytopathology 60:461-466.

25. Heesterbeek, J. A. P. 2002. A brief history of $R_{0}$ and a recipe for its calculation. Acta Biotheor. 50:189-204.

26. Jeger, M. J. 1982. The relation between total, infectious, and postinfectious diseased plant tissue. Phytopathology 72:1185-1189.

27. Jeger, M. J. 2000. Theory and plant epidemiology. Plant Pathol. 49:651658.

28. Jeger, M. J., and van den Bosch, F. 1993. Threshold criteria for model plant disease epidemics. II. Persistence and endemicity. Phytopathology 84:28-30.

29. Kato, H., and Kozaka, T. 1974. Effect of temperature on lesion enlargement and sporulation of Pyricularia oryzae on rice leaves. Phytopathology 64:828-830.

30. Kendall, D. G. 1949. Stochastic processes and population growth. J. Roy.
Stat Soc. B 11:230-282.

31. Kermack, W. O., and McKendrick, A. G. 1927. A contribution to the mathematical theory of epidemics. Proc. Roy. Soc. A 115:700-721.

32. Lalancette, N., and Hickey, K. D. 1986. An apple powdery mildew model based on plant growth, primary inoculum, and fungicide concentration. Phytopathology 76:1176-1182.

33. Latta, G. E. 1974. Transform methods. In: Handbook of Applied Mathematics. C. E. Pearson, ed. Van Nostrand, London.

34. Leonard, K. J., and Mundt, C. C. 1984. Methods for estimating epidemiological effects of quantitative resistance to plant diseases. Theor. Appl. Genet. 67:219-230.

35. Lewontin, R. C. 1965. Selection for colonizing ability. Pages 77-91 in: Genetics of Colonizing Species. H. G. Baker and G. L. Stebbins, eds. Academic Press, London New York.

36. Lotka, A. J. 1907. Studies on the mode of growth of material aggregates. Am. J. Sci. Ser. 4 Vol. 24:199-216.

37. Lotka, A. J. 1922. The stability of the normal age distribution. Proc. Natl. Acad. Sci. USA 8:11:339-345.

38. Lotka, A. J. 1928. The progeny of a population element. Am. J. Epidemiol. 8:6:875-901.

39. Lotka, A. J. 1929. Biometric functions in a population growing in accordance with a prescribed law. Proc. Natl. Acad. Sci. USA 15:10:793-798.

40. Lotka, A. J. 1939. On an integral equation in population analysis. Ann. Math. Stat. 10:2:144-161.

41. Lotka, A. J. 1942. The progeny of an entire population. Ann. Math. Stat. 13:117-118.

42. Madden, L. V. 2006. Botanical epidemiology: Some key advances and its continuing role in disease management. Eur. J. Plant Pathol. 115:3-23.

43. McCallum, H., Barlow, N., and Hone, J. 2001. How should pathogen transmission be modelled? Trends Ecol. Evol. 16:295-300.

44. McGregor, A. J., and Manners, J. G. 1985. The effect of temperature and light intensity on growth and sporulation of Puccinia striiformis on wheat. Plant Pathol. 34:263-271.

45. Meats, A. 1971. The relative importance to population increase of fluctuations in mortality, fecundity and the time variables of the reproductive schedule. Oecologia 6:223-237.29.

46. Mehta, Y. R., and Zadoks, J. C. 1977. Uredospore production and sporulation period of Puccinia recondita $\mathrm{f}$. sp. triticina on primary leaves of wheat. Neth. J. Plant Pathol. 76:267-276.

47. Metz, J. A. J., and Diekmann, O. 1986. The Dynamics of Physiologically Structured Populations. Lecture Notes in Biomathematics 68. SpringerVerlag, Berlin

48. Mortensen K., and Green G. J. 1978. Assessment of receptivity and uredospore production as components of wheat stem rust resistance. Can. J. Bot. 56:1827-1837.

49. Onstad, D. W., and Kornkven, E. A. 1992. Persistence and endemicity of pathogens in plant populations over time and space. Phytopathology 82:561-566.

50. Roberts, M., and Heesterbeek, H. 1993. Bluff your way in epidemic models. Trends Microbiol. 1:343-347.

51. Savary, S., De Jong, P. D., Rabbinge, R, and Zadoks, J. C. 1990. Dynamic simulation of groundnut rust: A preliminary model. Agric. Syst. 32:113141.

52. Segarra, J., Jeger, M. J., and van den Bosch, F. 2001. Epidemic dynamics and patterns of plant diseases. Phytopathology 91:1001-1010.

53. Teng, P. S., and Close, R. C. 1978. Effect of temperature and uredinium density on urediniospore production, latent period, and infectious period of Puccinia hordei Otth. N. Z. J. Agric. Res. 21:282-296.

54. van den Bosch, F., Frinking, H. D., Metz, J. A. J., and Zadoks, J. C. 1988. Focus expansion in plant disease. III: Two experimental examples. Phytopathology 78:919-925.

55. van den Bosch, F., Zadoks, J. C., and Metz, J. A. J. 1988. Focus expansion in plant disease. I: The constant rate of focus expansion. Phytopathology 78:54-58.

56. van den Bosch, F., Zadoks, J. C., and Metz, J. A. J. 1988. Focus expansion in plant disease. II: Realistic parameter-sparse models. Phytopathology 78:59-64.

57. Vanderplank, J. E. 1963. Plant Disease: Epidemics and Control. Academic Press, Inc., New York.

58. Vanderplank, J. E. 1965. Dynamics of epidemics of plant disease. Science 147:120-124.

59. Waggoner, P. E. 1986. Progress curves of foliar diseases: Interpretation and use. In: Plant Disease Epidemiology. K. J. Leonard and W. E. Fry, eds. MacMillan Publishing Co., New York.

60. Zadoks, J. C. 1971. Systems analysis and the dynamics of epidemics. Phytopathology 61:600-610. 